\title{
Design of Teaching Resource Management Platform Based on MVC
}

\author{
Hao Rao ${ }^{\mathrm{a}, *}$, Yonglun Gu ${ }^{\mathrm{b}}$ \\ School of Information Science and Engineering, Shaoguan University, Shaoguan, China \\ adrh999@163.com, b 112148650@qq.com
}

\begin{abstract}
The teaching resource management platform based on MVC mode was designed by PHP and MySQL to combine Internet technology with traditional teaching. The platform includes three modules: online learning, resource management and user management. It realizes user online video learning and information exchange, and uploading and downloading teaching resources, which fills the shortcomings of traditional teaching. The teaching process is flexible and autonomous, and the efficiency of teaching and the utilization of resources are improved.
\end{abstract}

Keywords: teaching resource, PHP, platform, MVC.

\section{Introduction}

The teaching resource management platform aims at providing a convenient online learning environment for teaching, and classifying, storing, searching and sharing huge teaching resources. With the combination of Internet technology and traditional teaching, the shortcomings of traditional teaching are made up to make teaching more efficient and the teaching resources are fully utilized.

PHP technology is used in the teaching resource management platform. The platform has three functions: online learning, resource sharing and user communication. Online learning is conducive to the improvement of teaching and learning efficiency. The sharing function of teaching resources makes teaching resources easy to share and maximize efficiency. The system also makes learning more practical and more efficient. The platform has certain positive significance, convenient learning and convenient management. Under the background of information age, this platform undoubtedly provides great convenience for teaching and learning.

\section{System function analysis}

The MVC framework is used in this system, and ThinkPHP is used in the PHP framework. ThinkPHP is an object oriented lightweight PHP development framework that follows the Apache2 open source protocol. Using ThinkPHP, it is more convenient and quick to develop and deploy WEB applications. After the user sends a request, ThinkPHP enters the ThinkPHP's MVC mode support logic after the module is explained. The module corresponds to the MVC's Controller controller. The controller performs the business logic, gets the data structure model, and determines the response View. Then the view template engine is invoked, and the response interface is finally returned to the customer.

Teaching resources management platform system to achieve three main functions: (1) the function of online learning, namely the user can study online video, can conveniently inquire into their required courses; (2) the resource sharing function, namely, resource search function, to provide resources to download; (3) user communication function, users can communicate, each comment on the platform.

Online learning module supports online video teaching. After teachers upload teaching video, students can choose their favorite direction for online learning. The online learning module also contains information communication modules. At the same time, it also provides courseware 
downloading. Before the user enters the online learning page, it is necessary to choose the Department, the professional selection, the subject selection, and the final choice of the course to watch the teaching video. Before watching the video, the user also needs to log in to the account.

Resource sharing module provides the function of resource search and download. Teaching related resources are uploaded by teachers. The focus of this module is the design of the search algorithm. The search algorithm design requires that the search speed is faster and the search results are sorted correctly.

Each account has user communication function, and all the users' rights have a view to review, modify the data, and view the historical function of video browsing. In addition, there are 2 functions of the teacher's user role: the teacher's personal document center and the course publishing center. The personal file center of the teacher is the server providing a free space for the teacher to store the video files and courseware uploaded. The course publishing center is a function provided by the server to a teacher for the release of a course. After uploading the files in the personal file center, the main teachers can add new courses to the curriculum publishing center, then select video files in each course, and each course can also be provided with some courseware to provide students downloading.

\section{Design of key modules}

The main purpose of the platform is to realize the exchange and sharing of teaching resources. The technologies required for the design platform include: PHP, MySQL, MVC framework, ThinkPHP, Ajax, web programming language and so on. The main functional modules are described as follows.

\subsection{Design of online learning module}

All the video on the platform is uploaded by the teacher. All videos are stored in file mode in the server. The database table saves the storage address of the file. When playing the video, read the file URL from the database, and the browser gets URL to play the video. Use HTML5's video tag for online video playback.

In order to show good animation effects, the module uses a large number of CSS and JavaScript code. Users browse the online learning module, and you can see the selection page of the learning module. Each learning module is displayed by a div. When the mouse is moved to div, the specific classification will be displayed, and clicking on the selected classification is a successful choice. Click the switch button to switch to the list mode, which can browse a large number of learning classification information at the same time.

After the choice of specific classification, that is to enter the subject and course selection page. The detailed list of subjects in the page is unexpanded at the beginning, when the user clicks on the subject Div. After the detailed list is expanded, the user can choose the teacher's course, check the subject introduction, and click the button to recover the detailed list. When you click on a course, you can get into the video playback page. The code of the page's controller is as follows.

public function index(\$chapterId=-1) \{

if(checkLogin()==0) \{\$this->error("Please Login",U('Home/Index/index'),2);

\$chapter=D('Chapter');

\$thisChapter=\$chapter-> where('state=0 AND id='.\$chapterId)->select();

if(! \$thisChapter)\{ \$this->error("choose again",U('Home/Course/index'),2); \}

\$this->assign("chapterId",\$chapterId); \$login_user=I('cookie.login_user','0');

\$username=session(\$login_user); \$user=D('user');

\$result=\$user-> where("username="'.\$username."'")->select();

\$userId=\$result[0]['id']; \$browserHistory=D('browserhistory');

\$data['user_id']=\$userId; \$data['chapter_id']=\$chapterId;

\$data['time']=date('y-m-d,H:i:s',time());

if(\$browserHistory->create(\$data))\{ \$browserHistory->add(); \}

\$this->assign("chapterName",\$thisChapter[0]['name']);

\$subjectId=\$thisChapter[0]['subject_id']; 
\$subject=D('Subject');

\$thisSubject=\$subject-> where('id='.\$subjectId)->select();

\$this->assign("subjectName",\$thisSubject[0]['name']);

\$teacherId=\$thisChapter[0]['user_id'];

\$user $=\mathrm{D}($ 'User');

\$thisTeacher=\$user-> where('id='.\$teacherId)->select();

\$this->assign("teacherName",\$thisTeacher[0]['name']);

\$chapterList=\$chapter-> where('state $=0 \quad$ AND

user_id='.\$teacherId.'

AND subject_id='.\$subjectId)->select();

\$this->assign("chapterList",\$chapterList);

\$file=D('File');

\$result=\$file->relation('Fcrelation')->select();

\$videoUrl=null;

foreach (\$result as \$key) \{

if(\$key['Fcrelation']\&\&\$key['type']=='mp4')\{

foreach (\$key['Fcrelation'] as \$key2) \{

if(\$key2['chapter_id']==\$chapterId) \{

\$videoUrl=\$key['url']; \}\}\}\}

\$this->assign('videoUrl',\$videoUrl);

\$result $=\$$ file- $>$ relation('Fcrelation')-> select();

\$downloadFileList=null;

$\$ \mathrm{i}=0$;

foreach (\$result as \$key) \{

if(\$key['Fcrelation']\&\&\$key['type']!='mp4')\{

foreach (\$key['Fcrelation'] as \$key2) \{

if(\$key2['chapter_id']==\$chapterId)\{

\$downloadFileList[\$i]['id']=\$key['id'];

\$downloadFileList[\$i]['type']=\$key['type'];

\$downloadFileList[\$i]['name']=\$key['name'];

$\$ \mathrm{i}++;\}\}\}\}$

\$this->assign('downloadFileList',\$downloadFileList); \$message=D('Message');

\$count $=$ \$message- $>$ count ()$; \quad$ \$page $=$ new Page $($ \$count, 5);

\$messageBlockList=\$message->relation('Reply')-> where('chapter_id='.\$chapterId)->limit(\$page

->firstRow.','.\$page->listRows)->select();

\$userList=\$user->field('id,name,headimage')->select();

\$this->assign("userList",\$userList);

\$show = \$page->show();

\$this->assign('messageBlockList', \$messageBlockList);

\$this->assign('page', \$show);

\$this->display('LearningOL/learning'); \}

\subsection{Design of information communication module}

The purpose of this module is to enable students to ask questions directly after studying online, and to ask questions directly on the current page. Any user can see that any user can answer this question to achieve the purpose of user communication.

The module is embedded in the video playback page, which can be regarded as the communication center of each subject. Because of the huge amount of information exchanged between users, the database of the information exchange module needs to be better designed, and the speed of the query needs to be faster.

Module design is similar to the way of forum communication. It can be reevaluated in others' comments. Therefore, the reply target of comment information should be stored in the data table. When the mark is 0 , the comment is the first commentator, not the respondent. When the mark is $n$ $(\mathrm{n}>0)$, it indicates that the comment is a comment that replies to the information of article $\mathrm{n}$. The 
module uses JavaScript code to switch on the client side, and Ajax technology is needed when data is updated. The code for sending information functions is as follows.

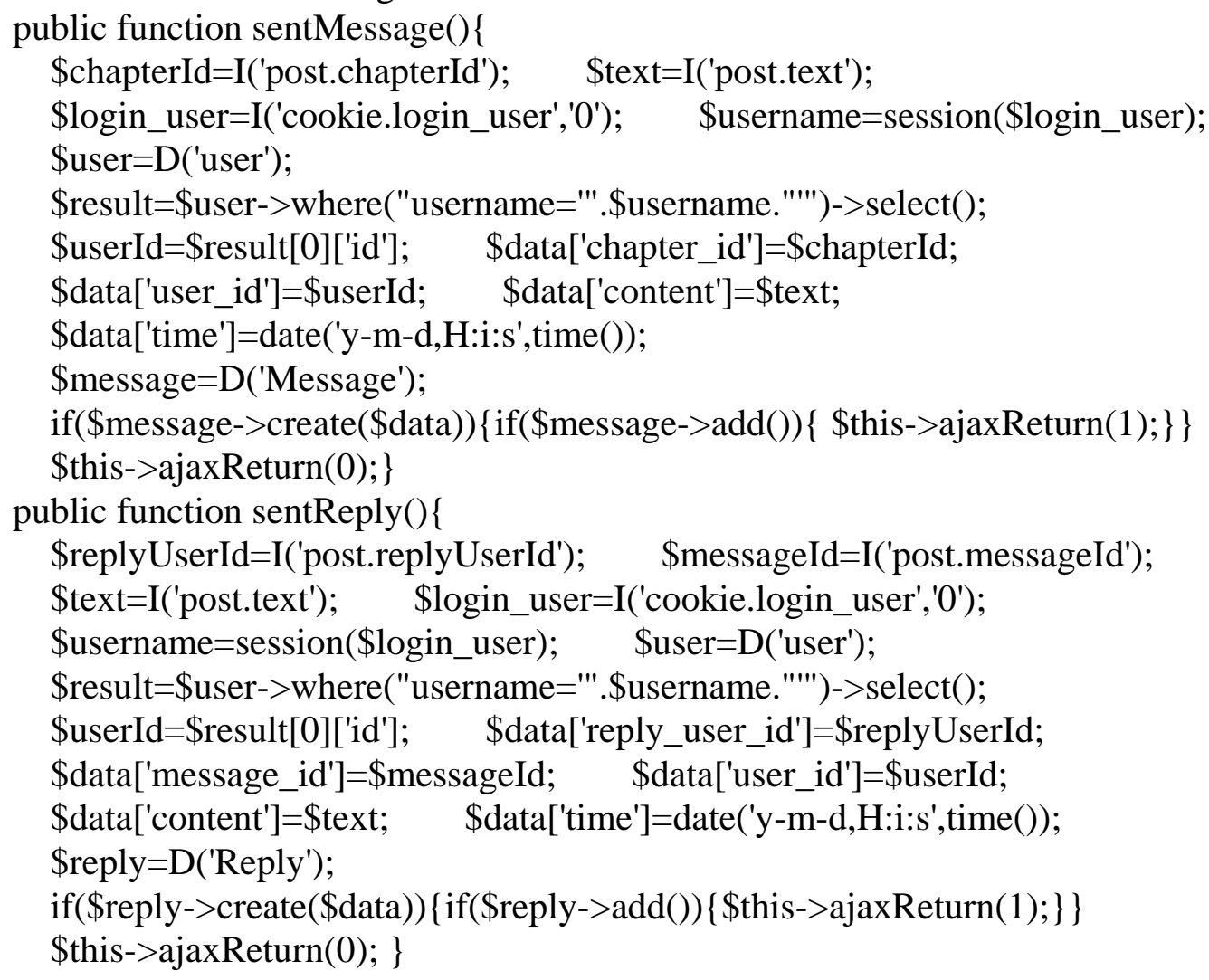

\section{Conclusions}

This paper discusses the development of a platform that can be combined with practical teaching. The platform can be combined with teachers' teaching plan, assisting teachers' teaching, and promoting students' interest and efficiency in learning. In order to improve the efficiency of teaching, it is convenient to manage, and the operation of the platform is simple and easy to understand, and the cost of development is low.

\section{References}

[1] FAN Guimei,HUANG Haijun.Scenario-Based Stochastic Resource Allocation with Uncertain Probability Parameters[J].Journal of Systems Science \& Complexity,2017,30(02):357-377.

[2] Bendong Zhao,Huanzhang Lu,Shangfeng Chen,Junliang Liu,Dongya Wu.Convolutional neural networks for time series classification[J].Journal of Systems Engineering and Electronics,2017,28(01):162-169.

[3] Zhigang Zou,Wangfang Che,Fuling Mu,Yiqian Chao,Bo Zhang.Role-based approaches for operational task-resource flexible matching model and algorithm[J].Journal of Systems Engineering and Electronics,2017,28(01):67-80.

[4] 4.QI Hongsheng,WANG Yuanhua,LIU Ting,CHENG Daizhan.Vector Space Structure of Finite Evolutionary Games and Its Application to Strategy Profile Convergence[J].Journal of Systems Science \& Complexity,2016,29(03):602-628.

[5] 5.Xiaolong Xu,Jinglan Zhou,Xinheng Wang,Yun Zhang.Multi-authority proxy re-encryption based on CPABE for cloud storage systems[J].Journal of Systems Engineering and Electronics,2016,27(01):211-223. 